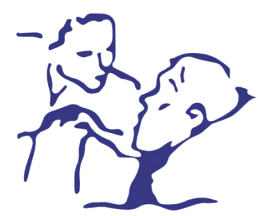

Medicina Paliativa

www.medicinapaliativa.es

ORIGINAL

\title{
Exploración de los valores y deseos de pacientes con enfermedad crónica avanzada y con enfermedad crónica compleja. Conversaciones acerca del final de la vida
}

\author{
Christian Villavicencio-Chávez ${ }^{* 1}$, Cristina Garzón-Rodríguez ${ }^{2}$, Jesús Vaquero-Cruzado ${ }^{3}$, \\ Enric Grácia ${ }^{4}$, Anna Torrents ${ }^{4}$ y Pilar Loncán ${ }^{5}$
}

\begin{abstract}
${ }^{1}$ Equipo de Cuidados Paliativos, Caredoctors, Barcelona. Programa LagunAdvance, Hospital Centro de Cuidados Laguna, Madrid. Facultad de Medicina, Universidad Internacional de Catalunya, Barcelona. 'Equipo de Cuidados Paliativos, Caredoctors. Servicio de Cuidados Paliativos, Institut Català d'Oncología, Barcelona. ${ }^{3}$ Equipo de Cuidados Paliativos, Caredoctors. Equipo de Gestión de la Cronicidad del Baix Llobregat Centre, Barcelona. ${ }^{4}$ Unidad de Cuidados Paliativos, Fundació Sociosanitaria Santa Susanna, Caldes de Montbui, Barcelona. ${ }^{5}$ Programa de Atención domiciliaria Equipo de Soporte (PADES), Fundació Sociosanitaria Santa Susanna, Caldes de Montbui, Barcelona
\end{abstract}

Recibido el 6 de septiembre de 2017

Aceptado el 19 de diciembre de 2017

\section{PALABRAS CLAVE \\ Planificación \\ anticipada de \\ cuidados, Cuidados \\ Paliativos, anciano \\ frágil.}

\begin{abstract}
Resumen
Objetivo: Explorar los deseos y valores de los pacientes con Enfermedad Crónica Avanzada (MACA) y Enfermedad Crónica Compleja (PCC), usando el Go Wish Card Game (GWG) como instrumento para establecer una conversación sobre el final de la vida.

Material y métodos: Se incluyeron pacientes MACA y PCC ingresados en la unidad de convalecencia y larga estancia de la Fundació Sociosanitaria Santa Susanna. Se registraron datos sociodemográficos, nivel de instrucción, nivel de competencia mediante la Escala Móvil de Drane (EMD) y estado cognitivo mediante la escala de Pfeiffer. Se usó el GWG como instrumento para explorar los deseos y valores. Los pacientes seleccionaron entre las cartas lo importante y no importante. De las importantes, se les pidió que seleccionaran las 10 más importantes. Se evaluó la utilidad y adecuación de la entrevista.

Resultados: Se incluyeron 16 mujeres y 7 hombres, la edad promedio fue de $82,39 \pm 7,38$. MACA: $13 \%$; PCC: 87 \%. El grado de instrucción fue: sin estudios: 56,5\%, estudios primarios: 43,5\%. Los niveles de competencia según la EMD fueron: I $(8,7 \%)$, II $(52,2 \%)$ y III $(39,1 \%)$. Pfeiffer $\leq 2$ errores: 60,8 \%; entre 3-4 errores: 30,4\%; entre 5-7 errores: 8,6\%. Los deseos identificados como más importantes fueron: "No ser una carga para mi familia" $(91,3 \%)$, "No estar conectado a máquinas" (69,6\%) y "No tener dolor" (69,6\%). Los deseos menos importantes fueron:
\end{abstract}


"Saber qué cambios puede tener mi cuerpo" $(73,9 \%)$; "Ser consciente de lo que está pasando" (65,2 \%); y "Morir en casa" (65,2 \%). La entrevista fue útil en el 91,3\% y apropiada en el 95,7\%.

Conclusión: No ser una carga para mi familia, no estar conectado a las máquinas y no tener dolor, fueron los deseos más importantes los pacientes MACA y PCC. El GWG fue un buen instrumento para explorar los deseos y valores acerca del final de la vida.

\section{KEYWORDS}

Advance care planning, palliative care, frail elderly.

Villavicencio-Chávez C, Garzón-Rodríguez C, Vaquero-Cruzado J, Grácia E, Torrents A, Loncán P. Explotación de los valores y deseos de pacientes con enfermedad crónica avanzada y con enfermedad crónica compleja. Conversaciones acerca del final de la vida. Med Paliat. 2019;26(1):43-49.

\section{Introducción}

El conocimiento de los valores y deseos de los pacientes por parte del personal sanitario ayuda a mejorar la atención médica, sobre todo en situaciones de edad avanzada ${ }^{1}$. Conocer estos valores y deseos permitirían la realización de una adecuada Planificación Anticipada de Cuidados (PAC) y, por tanto, contribuirían a mejorar el abordaje médico al final de la vida².

Explorar de manera precoz cuáles son estos valores garantiza el respeto a la autonomía de los pacientes cuando estos, en algunos casos, pierden la capacidad de decidir. Proporciona un conocimiento sobre la toma de decisiones, que es fundamental para el paciente y su familia en la etapa final de la vida ${ }^{3}$, evitando en algunos casos tratamientos invasivos y desproporcionados que generan mayor malestar emocional para ambos ${ }^{4}$.

La literatura evidencia distintas formas de exploración, especialmente con estudios cualitativos ${ }^{5,6}$, y además de otros instrumentos como el Go-Wish Card, que fue desarrollada por Coda Alliance en California en el año 2007 para facilitar conversaciones, frente a una hipotética situación de enfermedad grave , $^{2,-9}$ y explorar los deseos de los pacientes y permitir realizar una adecuada PAC con los pacientes con enfermedad avanzada.

En nuestro entorno existen pacientes identificados como pacientes con enfermedad crónica compleja (PCC) y pacien- tes con enfermedad crónica avanzada (MACA: Malaltia Crònica Avançada [término catalán empleado en los trabajos originales] $\left.{ }^{10,11}\right)$. Los PCC tienen una prevalencia que oscila entre un 3,5 y un $5 \%$ de la población. Estos pacientes se caracterizan por presentar pluripatología, con una evolución muy dinámica y alta utilización de servicios de urgencias, elevado consumo de recursos sanitarios, fármacos y necesidad de gestionar diferentes dispositivos para su atención ${ }^{5-8}$. Los MACA presentan una prevalencia que está alrededor del 1,5\% de la población, con un perfil clínico equivalente al PCC (enfermedad crónica progresiva y evolutiva) pero, además, identificación por parte de los profesionales de una afectación clínica de más gravedad, comparado con el estándar de las personas con las mismas enfermedades y condiciones, con una percepción de un pronóstico de vida limitado ${ }^{12,13}$.

La exploración de los valores y posterior desarrollo de la PAC se ha propuesto como una manera de permitir que los pacientes en situación de enfermedad avanzada puedan expresar sus voluntades, valores y preferencias cuando se encuentren ante un proceso irreversible y de mal pronóstico?.

Para realizar una adecuada PAC es importante abordar el tema en estadios precoces de la evolución de la enfermedad terminal, De Vleminck y cols. sugieren, además, que debería ser iniciado en atención primaria ${ }^{14}$. 
Uno de los factores más importantes en la PAC es conseguir una adecuada comunicación con el enfermo que permita, por un lado, transmitir una información adecuada sobre su enfermedad, y por otro lado tener en cuenta el mundo de valores del paciente, para que pueda participar directamente en la toma de decisiones sobre su proceso ${ }^{15,16}$. Esto hace imprescindible una adecuada formación de los profesionales en actitudes, conocimientos y habilidades en el proceso de comunicación ${ }^{17,18}$.

En este trabajo presentamos la implementación del Go Wish Cards ${ }^{2,7-9}$. Ya que la exploración de valores y deseos de los pacientes con enfermedad avanzada no es habitual en nuestro medio, este estudio se plantea como objetivo explorar los valores y deseos de los pacientes con enfermedad crónica compleja y pacientes con enfermedad crónica avanzada frente a una situación irreversible y de mal pronóstico.

\section{Método}

Estudio prospectivo descriptivo. Se incluyeron de manera consecutiva todos los pacientes que ingresaron en el centro sociosanitario durante el periodo de estudio, desde julio a octubre de 2016, que cumplieran los criterios de inclusión y que firmaran el consentimiento informado. Pacientes con enfermedad crónica compleja (PCC) y pacientes con enfermedad crónica avanzada (MACA ${ }^{10}$ ingresados en la unidad de media y larga estancia de la Fundació Socio-sanitaria Santa Susanna.

Los criterios de inclusión fueron: pacientes capaces de comunicarse adecuadamente con el personal de investigación, adecuada capacidad de comprensión y comunicación, estar informados de que su enfermedad es crónica e irreversible y firmar el consentimiento informado. Los criterios de exclusión fueron: pacientes con trastorno psiquiátrico, así como aquellos cuyo estado físico, cognitivo o psicológico que según el criterio médico sea demasiado frágil para permitir su inclusión.

Este estudio ha sido evaluado y aprobado por el Comité de Investigación Clínica del Instituto Universitario de Investigación en Atención Primaria Jordi Gol (IDIAP) y el Comité de Ética Asistencial del Valles Oriental (CEAVOC).

\section{Variables}

Se recogieron la edad, sexo, diagnóstico principal y grado de instrucción de todas las personas entrevistadas.

Las variables incluidas en este estudio fueron:

- El Instrumento NECPAL ${ }^{12}$ : fue utilizado para clasificar a los pacientes en PCC y MACA. Es un instrumento para la identificación de personas en situación de enfermedad avanzada-terminal y necesidad de atención paliativa en servicios de salud y sociales. Consta de cuatro preguntas. Se considera positivo si la pregunta 1 (pregunta sorpresa) "¿Le sorprendería que este paciente muriese en los próximos 12 meses?" es con respuesta negativa, y al menos otra de las siguientes tres preguntas acerca de la elección o demanda de cuidados paliativos, la presencia de indicadores clínicos generales de severidad y progresión e indicadores clínicos de severidad y progresión por patología, con respuesta positiva.
- Escala Móvil de Drane ${ }^{19}$ : Desarrollada para clasificar la capacidad de decidir según criterio subjetivo del entrevistador, consta de tres niveles: nivel I: se sitúan las decisiones de consentimiento a intervenciones de elevado beneficio y bajo riesgo para el paciente; nivel II: se encuentran las decisiones de consentimiento o rechazo a las intervenciones médicas de eficacia dudosa; nivel III: en este nivel se hallan las decisiones de rechazar las intervenciones médicas de alto beneficio y de bajo riesgo, o consentir a tratamientos de alto riesgo y bajo beneficio. Por ejemplo, los pacientes con un nivel I en la escala móvil de Drane son los que se consideran con capacidad para decisiones clínicas sencillas, como realizar un análisis de sangre para un seguimiento de algún proceso crónico. Los pacientes que se encuentren dentro del nivel III son los que presentan mayor capacidad para decidir frente a decisiones de alta complejidad, como rechazar un procedimiento después de haber realizado un análisis de la situación.

De hecho, Drane habla de estos tres niveles o grados de dificultad de las decisiones. En el nivel I, el de menor dificultad, la mayor parte de las personas serán consideradas capaces, mientras que en el nivel III, que implica alta complejidad de las decisiones, muchas personas serán consideradas incapaces ${ }^{19,20}$.

- SPMSQ de Pfeiffer ${ }^{21}$ : cuestionario heteroadministrado que consta de 10 ítems. El punto de corte está en tres o más errores, en el caso de personas que al menos sepan leer y escribir, y de cuatro o más para los que no. A partir de esa puntuación existe la sospecha de deterioro cognitivo.

- Para evaluar los deseos y valores de los pacientes PCC y MACA $^{10}$ se utilizó el Go Wish Card ${ }^{2,7-9}$ : instrumento diseñado por Coda Alliance para generar una oportunidad para pensar en cómo desea ser tratado si enferma gravemente. Consiste en 35 cartas y una carta libre; cada carta tiene un tema que puede ser importante a una persona enferma o que esté en situación paliativa.

\section{Procedimiento y recogida de variables}

En primer lugar, se realizó una entrevista informativa a aquellos pacientes que cumplían criterios de inclusión y, por lo tanto, eran considerados candidatos a participar en el estudio. Se les explicó las características y objetivos del estudio, se aclararon las posibles dudas y se les solicitó firmar el consentimiento informado, en caso de que quisieran participar.

Se procedió a la clasificación de pacientes en pacientes PCC y MACA $^{10}$ mediante el instrumento NECPAL ${ }^{12}$.

Se registraron los niveles de capacidad de comprensión mediante la escala móvil de Drane ${ }^{19}$ y el estado cognitivo mediante la escala de Pfeiffer ${ }^{21,22}$.

Se procedió a realizar una entrevista sobre los valores y deseos en caso de posibles complicaciones en su estado de salud, usando el Go Wish Card Game (GWC) $)^{2,7-9}$ como instrumento. Se le pidió al paciente que revisara las cartas que incluían valores y preferencias en una hipotética situación final de vida y las separara en dos grupos, separando cuáles eran importantes y nada importantes. Se le preguntó por otro tema importante que no se hubiese mencionado en las cartas anteriores y que le gustase comentar usando la carta 
libre. Seguidamente se le pidió que, de las importantes, seleccionara las 10 más importantes.

Finalmente se evaluó el grado de utilidad y de adecuación de la entrevista realizada mediante dos preguntas (Anexo 1).

\section{Análisis estadístico}

Se realizó un análisis descriptivo con frecuencia y porcentaje de las variables sociodemográficas; para las variables cuantitativas se calculó la media y desviación típica porque seguían una distribución normal. Los intervalos de confianza se calcularon con un $95 \%$ de nivel de confianza y los test con un nivel de significación del $5 \%$.

Para el análisis estadístico se utilizó el SPSS 21.0 para MAC.

\section{Resultados}

23 pacientes completaron la intervención. La edad media fue de 82,39 años (DE = 7,38). El 56,5\% (13) no tenía estudios, pero sabía leer y escribir; el 43,5 \% tenía estudios primarios. El diagnóstico principal de los pacientes al ingreso se muestra en la Tabla I.

El 69,6\% fueron mujeres y el 30,4\% varones. El $87 \%$ de los pacientes entrevistados fueron PCC y el $13 \%$ MACA.

Respecto a la clasificación de la escala móvil de competencia de Drane, el mayor porcentaje de los pacientes se encontraba situado en un Nivel 2 (Tabla II). La Tabla III muestra los porcentajes de deterioro cognitivo evaluados mediante el cuestionario de Pfeiffer.

Los diez valores y deseos más importantes mencionados por los pacientes, así como los diez menos importantes, se muestran en las Tablas IV y V, respectivamente.

Respecto a la evaluación final de la utilidad y adecuación de la entrevista, los porcentajes de respuesta se muestran en la Tabla VI.

Tabla I. Diagnóstico principal al ingreso

\begin{tabular}{lc}
\hline & $\%(\mathrm{n})$ \\
\hline Enfermedad pulmonar obstructiva crónica & $30,4(7)$ \\
Insuficiencia renal & $21,7(5)$ \\
Diabetes mellitus & $21,7(5)$ \\
Arritmia cardiaca & $13,1(3)$ \\
Insuficiencia cardiaca & $13,1(3)$ \\
\hline
\end{tabular}

Tabla II. Escala móvil de competencia de Drane

\begin{tabular}{cc}
\hline Nivel I & $8,7 \%$ \\
Nivel II & $52,2 \%$ \\
Nivel III & $39,1 \%$ \\
\hline
\end{tabular}

Tabla III. Errores en el cuestionario de Pfeiffer

\begin{tabular}{cc}
\hline Valor & $\%(\mathrm{n})$ \\
\hline $0-2$ & $60,8 \%(14)$ \\
$3-4$ & $30,4 \%(7)$ \\
$5-7$ & $8,6 \%(2)$ \\
\hline
\end{tabular}

Tabla IV. Principales valores y deseos de los pacientes

\begin{tabular}{lc}
\hline \multicolumn{2}{c}{ Carta más importantes } \\
\hline \multicolumn{2}{c}{$\%$} \\
\hline No ser una carga para mi familia & 91,3 \\
No estar conectado a máquinas & 69,6 \\
Estar sin dolor & 69,6 \\
Tener a mi familia conmigo & 56,5 \\
Estar en paz con Dios & 56,5 \\
Mantener mi dignidad & 56,5 \\
Tener confianza en mi doctor & 39,1 \\
Hablar y ser comprendido & 39,1 \\
Poder ayudar a los demás & 39,1 \\
No morir solo & 34,8 \\
\hline
\end{tabular}

Tabla V. Valores y deseos menos importantes para los pacientes

\begin{tabular}{lc}
\hline \multicolumn{2}{c}{ Carta } \\
\hline \multicolumn{2}{c}{ menos importantes } \\
\hline Saber qué cambios puede tener mi cuerpo & 73,9 \\
Estar consciente & 65,2 \\
Tener mis asuntos financieros en orden & 65,2 \\
Morir en casa & 65,2 \\
Recordar logros personales & 65,2 \\
Atender asuntos inconclusos con mi familia y amigos & 60,9 \\
Hablar con un sacerdote, pastor, rabino... & 60,9 \\
Hablar de lo que significa morir & 69,6 \\
Poder estar en casa & 60,9 \\
Disfrutar de la comida & 56,5 \\
\hline
\end{tabular}

Tabla VI. Valoración final de la entrevista, entre utilidad y adecuación de la entrevista

\begin{tabular}{ccc}
\hline & Útil \% (n) & Adecuado \% (n) \\
\hline Nada & $0(0)$ & $0(0)$ \\
Un poco & $0(0)$ & $4,3 \%(1)$ \\
Bastante & $8,7 \%(2)$ & $0(0)$ \\
Mucho & $56,7 \%(13)$ & $60,9 \%(14)$ \\
Muchísimo & $34,8 \%(8)$ & $34,8 \%(8)$ \\
\hline
\end{tabular}




\section{Discusión}

La idoneidad de la atención al final de la vida y la realización de una adecuada PAC requieren una exploración de los valores y deseos de los pacientes de forma individual ${ }^{8}$. Son cada vez más frecuentes los trabajos que hablan de la PAC usando el GWC ${ }^{2,8,9}$, y existen algunos estudios cualitativos, como los de Downey y cols. en Whashington ${ }^{5}$ y de Steinhauser y cols. en Durham. ${ }^{6}$, en los que se realizan exploraciones en profundidad sobre las preferencias y deseos de los pacientes.

Como muestra la literatura, el GWC ${ }^{2,8,9}$ es un instrumento utilizado para evaluar los valores y preferencias de los pacientes frente a una situación amenazante para la vida y podría abrir una puerta a la PAC; además ha sido implementado en diferentes poblaciones, tanto con pacientes con cáncer ${ }^{2}$ como pacientes con patología mixta ${ }^{8,9}$.

La población seleccionada en este estudio es una población de pacientes con enfermedades crónicas complejas y pacientes con enfermedades crónicas avanzadas, en este caso, el GWC ${ }^{2,8,9}$ ha sido el instrumento utilizado para la exploración de valores y deseos de los pacientes y servir de ayuda en la PAC.

En el caso de la población a estudio, podemos observar que después de realizar la entrevista, dentro de los principales deseos y valores de los pacientes, un alto porcentaje de la población $(91,3 \%)$ manifestó que: "No ser una carga para su familia", se encontraba dentro de sus deseos más importantes, resultado que contrasta con otros estudios como los de Lankarani y cols. ${ }^{8}$ y Delgado-Guay y cols. ${ }^{2}$ usando el GWC $2,8,9$, donde se encontraba en un quinto lugar para ambos con un $45,5 \%$ y un $49 \%$ de preferencia, respectivamente. Una posible explicación podría ser que nuestros pacientes ya estaban ingresados en un centro sociosanitario, y que probablemente esta situación les ha hecho ver que, a diferencia de su domicilio, sus necesidades de cuidado están cubiertas por otros profesionales y no directamente por la misma familia. Es por esto que lo manifiestan como un deseo muy importante en este nuevo contexto.

"Estar sin dolor" se encontraban en una segunda posición dentro de las preferencias de nuestros pacientes, con un $69,6 \%$. Muestra similitud con el estudio realizado por Lankarani y cols. en los Ángeles, donde mostró que estar sin dolor se encontraba dentro de los deseos más importantes de sus pacientes en un $60 \%$, seguido de "Estar en paz con Dios" y "Rezar/orar" en un 51,5\% y 45,5\%, respectivamente. La espiritualidad, sin embargo, en el estudio de Delgado-Guay resultó ser prioritario a "Estar sin dolor", lo que coincide con los estudios realizados por Litzelman y cols. en el domicilio", y en el caso del estudio realizado por Lankarani y cols. ${ }^{8}$ en pacientes hospitalizados. Esto permite concluir a los autores que, para un buen abordaje de la PAC, es necesario la exploración de estos temas y deberían ser considerados como factores contribuyentes para una buena muerte.

Existen otros estudios, como los de Downey y cols. ${ }^{5}$ en la Universidad de Washington y otro en la Universidad de Chicago por Steinhauser ${ }^{6}$, con pacientes con patología mixta avanzada (no oncológica y oncológica), que no usan el GWC ${ }^{2,8,9}$ pero que exploran los principales deseos de los pacientes dentro de un protocolo de investigación cualitativa, es decir, con entrevistas en profundidad. En estos estudios se eviden- cia que el dolor y el control de los síntomas se encuentran dentro de las principales preferencias de los pacientes con enfermedad terminal.

Respecto a "No estar conectado a máquinas", es una preocupación importante dentro de nuestra población con un porcentaje muy similar a "Estar sin dolor" (69,6\%), resultados parecidos a los de Kelley y cols. ${ }^{23}$, en la que muestra en la que un $73 \%$ de los pacientes no desean ventilación mecánica y un $81 \%$ no quieren intubación.

En relación con las 10 cosas menos importantes, hemos de destacar que "Saber qué cambios puede tener mi cuerpo" presentaba mayor puntuación, dentro de las 10 cosas menos importantes para los pacientes. Una probable explicación, según la tesis de Daniela Thumala ${ }^{24}$, es que las personas mayores durante el proceso de envejecimiento, se van acostumbrado a su pérdidas y el aspecto físico deja de ser relevante.

Encontramos muy útil la carta sobre "Estar consciente de lo que está pasando", ya que los pacientes en base a este enunciado comentaban que no querían ser conscientes, por lo que la escogían entre las menos importantes en un 65,2 \%. Pero nos permitió abordar el tema de la sedación paliativa y la posibilidad de disminución de la consciencia en caso de que la situación pronóstica empeorara y/o hubiera un síntoma refractario.

Dentro de nuestros resultados podemos observar que, si bien es cierto, "Estar en paz con Dios" como tema espiritual se encontraba dentro de los diez valores y deseos más importantes, no así el "Poder hablar con un sacerdote, pastor, rabino, capellán...". Una probable explicación, como ya se ha comentado anteriormente, es la necesidad espiritual de las personas con enfermedad avanzada, pero no así sobre la necesidad del soporte de una persona física para manifestar su espiritualidad.

Acerca del enunciado de "Morir en casa", hay estudios que muestran que para los pacientes el hecho de morir en casa puede ser un valor ${ }^{25-27}$; sin embargo, para nuestra población no fue así, probablemente debido a que ya se encontraban institucionalizados. Ya se ha iniciado la segunda parte del presente estudio que tiene como objetivo evaluar las preferencias y deseos en pacientes atendidos en su domicilio, lo que nos permitirá contrastar las dos muestras de población.

La carta número treinta y seis (Carta Libre) nos permitió hablar de algunos aspectos particulares al final de la intervención, que no se mencionaron durante la entrevista. En su gran mayoría se trataron temas como la incineración, el entierro y los detalles de la ceremonia.

Respecto al estado cognitivo, un objetivo secundario del estudio era evaluar el estado cognitivo y registrar la competencia de los sujetos. Para evaluar el estado cognitivo se utilizó la escala de Pfeiffer ${ }^{21}$, y para registrar la capacidad de decidir de los pacientes se decidió utilizar la escala móvil de Drane ${ }^{19}$, ya que algunos estudios muestran que para la toma de decisiones es importante evaluar la competencia, más que el estado cognitivo ${ }^{28}$. Incluso un paciente con demencia moderada, con capacidad cognitiva deteriorada, puede todavía ser capaz de indicar una elección y mostrar cierta comprensión ${ }^{29}$ en aspectos de baja complejidad ${ }^{19,30}$.

En la población estudiada, la gran mayoría de los pacientes se encontraba dentro del nivel II de la escala móvil de Drane $^{19}$, lo que nos hace ver que presentaban un grado de 
comprensión capaz de conocer la situación clínica y tratamiento médico, así como la capacidad de elección basada en las expectativas médicas ${ }^{19,30}$.

Además, según los resultados obtenidos, podemos observar que solo un $8,7 \%$ se encontraba dentro del nivel I, lo que podría coincidir con el estado cognitivo de alguno de ellos, cuyo valor en la escala de Pfeiffer fue entre 5-7 (8,6 \%), es decir, deterioro cognitivo moderado. Para evaluar y registrar la capacidad de decidir de los pacientes de la población a estudio se requirió una entrevista directa con el paciente, utilizando preguntas abiertas, incluyendo enfoques informales como formales, dependiendo de la situación y del contexto ${ }^{28}$. Hemos de aclarar que ningún paciente fue excluido por situación cognitiva y que ambas escalas, tanto la escala de Pfeiffer como la de Drane, han sido utilizadas para describir a nuestra población de estudio, ya que consideramos que la alteración cognitiva puede no excluir al paciente en su capacidad para decidir ${ }^{28}$.

\section{Limitaciones del estudio}

Aunque este estudio presenta las preferencias de valores y deseos de los pacientes con enfermedad crónica compleja y enfermedad crónica avanzada, tiene algunas limitaciones que necesitan ser consideradas. Los pacientes están ingresados en un centro sociosanitario, por lo que podría haber un sesgo en sus respuestas, ya que el hecho de que se encuentren institucionalizados podría influir en sus preferencias y valores. Además, puede existir el sesgo relacionado a la selección de la muestra, ya que son pacientes con una situación de comorbilidad concreta, lo que no haría extrapolables los resultados a otras poblaciones en un contexto clínico distinto.

\section{Conclusiones}

El GWC es un instrumento que permite explorar los deseos y valores de los pacientes de manera adecuada, facilitando aspectos comunicativos.

Las preferencias de nuestros pacientes se centraron principalmente en no ser una carga para su familia, así como la preocupación por el control sintomático y de no alargar innecesariamente la supervivencia.
Los aspectos espirituales son también temas de gran importancia para los pacientes que deben abordarse de manera adecuada al final de la vida.

\section{Conflicto de intereses}

Los autores declaran la ausencia de conflicto de intereses.

\section{Agradecimientos}

Quisiéramos agradecer a la Fundació Santa Susanna el permitirnos el desarrollo del estudio en sus instalaciones, y a la Cátedra We Care el análisis estadístico de esta parte del estudio.

\section{Bibliografía}

1. Ebenau A, Van Gurp J, Hasselaar J. Life values of elderly people suffering from incurable cancer: a literature review. Patient Educ Couns. 2017;100(10):1778-86.

2. Delgado-guay MO, Rodriguez-nunez A, Cruz V De, Frisbee-Hume $\mathrm{S}$, Williams J, Wu J, et al. Advanced cancer patients' reported wishes at the end of life : a randomized controlled trial. Support Care Cancer. 2016;24(10):4273-81.

3. Huang H, Chiu T, Lee L, Yao C, Chen C, Hu W. Family experience with difficult decisions in end-of-life care. Psychooncology. 2012;21(7):785-91.

4. Wright AA, Zhang B, Ray A, Mack JW, Trice E, Balboni T, et al. Associations between end-of-life discussions, patient mental health, medical care near death, and caregiver bereavement adjustment. JAMA. 2008;300(14):1665-73.

5. Downey L, Engelberg RA, Randall Curtis J, Lafferty WE, Patrick DL. Shared Priorities for the End-of-Life Period. J Pain Symptom Manage. 2009;37(2):175-88.

6. Steinhauser KE, Christakis NA, Clipp EC, et al. Preparing for the end of life: preferences of patients, families, physicians, and other care providers. J Pain Symptom Manage. 2001;22(3):727-37.

7. Menkin ES. Go Wish: a tool for end-of-life care conversations. J Palliat Med. 2007;10(2):297-303.

8. Lankarani-Fard A, Knapp H, Lorenz KA, Golden JF, Taylor A, Feld JE, et al. Feasibility of Discussing End-of-Life Care Goals with Inpatients Using a Structured, Conversational Approach: The Go Wish Card Game. J Pain Symptom Manage. 2010;39(4):637-43.

Anexo 1.

Considera útil la entrevista que le acabo de realizar

Nada Un poco Bastante Mucho Muchísimo

Considera adecuada la entrevista que le acabo de realizar

Nada Un poco Bastante Mucho Muchísimo 
9. Litzelman DK, Inui TS, Schmitt-Wendholt KM, Perkins A, Griffin WJ, Cottingham AH, et al. Clarifying Values and Preferences for Care Near the End of Life: The Role of a New Lay Workforce. J Community Health. 2017;42(5):926-34.

10. Gomez-Batiste X, Lasmarías C, González-Barboteo J, Calsina A, Amblas J, Contel JC, et.al. Reflexions sobre l'ètica en la identificació, el registre i la millora de l'atenció de les persones en situació crònica de complexitat clínica i malaltia avançada (PCC i MACA) 2013:1-28.

11. Gómez-Batiste X, Martínez-Muñoz M, Blay C, Amblàs J, Vila L, Costa X, et al. Proyecto NECPAL CCOME-ICO ${ }^{\circ}$ : Instrumento para la identificación de personas en situación de enfermedad crónica avanzada y necesidades de atención paliativa en servicios de salud y social. Centro Colaborador de la OMS para Programas Públicos de Cuidados Paliativos. Institut Català d'Oncologia. Accesible en: http: / / www.iconcologia.net.

12. Gómez-Batiste X, Martínez-Muñoz M, Blay C, Amblà J, Vila L, Costa X. Identificación de personas con enfermedades crónicas avanzadas y necesidad de atención paliativa en servicios sanitarios y sociales: elaboració $\mathrm{n}$ del instrumento NECPAL CCOMSICO. Med Clin. 2013;140(6):241-5.

13. Gómez-Batiste X, Martínez-Muñoz M, Blay C, Amblàs J, Vila $L$, Costa $X$, et al. Identifying patients with chronic conditions in need of palliative care in the general population: development of the NECPAL tool and preliminary prevalence rates in Catalonia. BMJ supportive \& palliative care. 2013;3(3):300-8.

14. De Vleminck A, Houttekier D, Deliens L, Stichele, Pardon K. Development of a complex intervention to support the initiation of advance care planning by general practitioners in patients at risk of deteriorating or dying:a phase $0-1$ study. BMC Palliat Care. 2016;15:1-10.

15. Strand JJ, Kamdar MM, Carey EC. Top 10 Things Palliative Care Clinicians Wished Everyone Knew About Palliative Care. Mayo Clin Proc. 2013;88(8):859-65.

16. Longo DL, Blinderman CD, Billings JA. Comfort Care for Patients Dying in the Hospital. N Engl J Med. 2015;373(26):2549-61.

17. Cripe LD, Frankel RM. Dying From Cancer: Communication, Empathy, and the Clinical Imagination. J Patient Exp. 2017;4(2):69-73.
18. Tanzi S, Biasco G, Baile W. Enhancing the Empathic Connection: Using Action Methods to Understand Conflicts in End-of-Life Care. J Patients Exp. 2014;1(1):14-9.

19. Drane JF. Competency to give an informed consent. A model for making clinical assessments. JAMA. 1984;252(7):925-7.

20. Simón-Lorda P. La capacidad de los pacientes para tomar decisiones: una tarea todavía pendiente. Rev la Asoc Española Neuropsiquiatría. 2008;28(2):327-50.

21. Martínez de la Iglesia J, Dueñas Herrero R, Onís Vilches MC, Aguado Taberné C, Albert Colomer C, Luque Luque R. [Spanish language adaptation and validation of the Pfeiffer's questionnaire (SPMSQ) to detect cognitive deterioration in people over 65 years of age]. Med Clin (Barc). 2001;117(4):129-34.

22. Pfeiffer E. A short portable mental status questionnaire for the assessment of organic brain deficit in elderly patients. J Am Geriatr Soc. 1975;23(10):433-41.

23. Kelley AS, Wenger NS, Sarkisian CA. Opiniones: End of Life Care Preferences and Planning Among Older Latinos. J Am Geriatr Soc. 2010;58(6):1109-16.

24. Daniela T, Dockendorff T, Yañez J, Santiago M. Formas de afrontamiento a las pérdidas asociadas al envejecer. Tesis doctoral. Universidad de Chile. 2011. http://repositorio.uchile.cl/tesis/ uchile/2011/cs-thumala_d/pdfAmont/csthumala_d.pdf.

25. Gomes B, Higginson IJ, Calanzani N, Cohen J, Deliens L, Daveson BA, et al. Preferences for place of death if faced with advanced cancer: a population survey in on behalf of PRISMA. Annals of oncology. 2012;23(8):2006-15.

26. Bell CL, Somogyi-Zalud E, Masaki KH. Methodological review: measured and reported congruence between preferred and actual place of death. Palliat Med. 2009;23(6):482-90.

27. Shepperd S, Dc G, Se S, Wee B. Hospital at home: home-based end-of-life-care. Cochrane Database Syst Rev. 2011;(7):CD009231.

28. Hegde S, Ellajosyula R. Capacity issues and decision-making in dementia. Ann Indian Acad Neurol. 2016;19(Suppl 1):S34-9.

29. Capacidad para tomar decisiones durante la evolución de una demencia: reflexiones, derechos y propuestas de evaluación. 2009. http://www.sen.es/pdf/2009/Documento_Sitges2009.pdf.

30. Vidal S. Competencia Para La Toma De Decisiones En La Práctica Clínica. Jurisprud Argentina. 1999;6166:58-68. http:// www.cba.gov.ar/wpcontent/4p96humuzp/2012/08/vidal.pdf. 